nitrate for examination in the field-ion microscope while the crystallographer will be irritated to read the word lattice where structure is meant. Nevertheless, the relatively high cost of the book will be amply repaid to anybody who intends to establish a field-ion microscope unit.

D. A. SMith

\section{MATRIX METHODS}

Matrix Methods

An Introduetion. By Richard Bronson. Pp. xiii +284 . (Academic Press: New York and London, January 1970.) $93 s$.

THIs book is designed for engineers and scientists in an American university. It has several good features in. cluding a large number of worked examples, a full set of solutions for the many problems (the solution to problem l.4.2 contains a slip, but no other slips were found in the high proportion tested), a step at a time approach to each new topic and warnings of pitfalls. The author concentrates on (theoretical) techniques and refers to several other books for many proofs. He pays lip service to numerical analysis but hardly mentions practical difficulties (for example, the impracticality of Cramer's rule is mentioned two pages after the ritual appearance of the rule, Gaussian elimination, without any discussion of the danger of zero, or nearly zero, pivots, is developed in a form which is easily understood but not suitable for practical use).

Some proofs are given for the case of $3 \times 3$ matrices but not all of these generalize easily. Some of those proofs could be made more rigorous without making them too difficult. The notation and terminology is fairly standard, but in some cases unnecessary difficulties are caused (for example, in finding inverses the matrix $\left[\begin{array}{l}2110 \\ 3401\end{array}\right]$ is written $\left[\begin{array}{c}21 \\ 34\end{array}\right]\left[\begin{array}{c}10 \\ 01\end{array}\right]$; commas are omitted in the matrix $\left.\left[x_{1}, x_{2}, \ldots, x_{n} \cdot\right]\right)$

The first four chapters establish the ususal elementary properties of matrices, determinants and linear equations. The last five chapters go some way towards justifying the book. The treatment of eigenvalues and vectors and generalized eigenvectors leading up to Jordan canonical forms should be easily understood. Methods of evaluating polynomials in a matrix $A$ and certain other functions of $A$ (mainly $\exp A$ and $\exp t A$ ) are clearly explained. (The reader should be on his guard when the sections on functions of $A$ are reached-the author twice gives the impression that any function $f(A)$ of a matrix exists, but the warning that $f$ is restricted comes several pages later in each case and could easily be missed.)

There is a good introduction to the use of $\exp A$ in the theoretical solution of a set of simultaneous linear differential equations and its adjoint system. The last chapter gives an adequate introduction to special classes of matrices (for example, Hermitian, Unitary, positive-definite).

F. W. Ponting

\section{CRACK CONFIGURATIONS}

Crack Problems in the Classical Theory of Elasticity By I. N. Sneddon and M. Lowengrub. (The SIAM Series in Applied Mathematics.) Pp. viii + 221. (Wiley: New York and London, March 1970.) $140 s$.

THIs book contains a comprehensive review of the analytical methods of solution of equilibrium crack problems for isotropic linear elastic bodies. Attention is focused primarily on "line" or Griffith cracks in two-dimensional problens and penny-shaped cracks in three-dimensional problems. For each crack configuration considered the authors describe and compare the various analytical appreaches which have led to solutions with appropriate references.
The single Griffith crack in an infinite plate under various loadings is first discussed. The solutions are then generalized to cope with the inflation of two equal cracks, an infinite row of collinear cracks, an infinite row of parallel cracks, and a crack in a strip with stress free edges. Other more awkward two-dimensional problems considered are those of a crack perpendicular to the edge of a semi-infinite elastic plate, and of radial cracks originating at the boundary of an internal circular hole in an infinite plate. The equivalent three-dimensional problems involving the inflation of penny-shaped cracks in an infinite medium, in a thick plate, and in a long circular cylinder are investigated in dotail. Other loadings such as the shear and torsion of a penny-shaped crack in an infinite medium and the effect of such a crack on the thermal stresses in an infinite medium are also discussed. A rather brief account is given of the Griffith criterion for failure, and this is used to estimate the critical internal pressure or external tension at which the cracks might be expected to grow. The stress concentration factors are also evaluated. A brief discussion is included of Barenblatt's approach concerning cohesive stresses at the tips of a crack. A short section reviews some of the work on propagating cracks and the onset of branching.

The book is devoted entirely to the analytical methods of solution of the boundary value problems posed by cracks in linearly elastic bodies. As such it is a very valuable survey of the literature in this extensive field and contains a useful and comprehensive bibliography.

Inevitably when this amount of material is condensed the demands on the reader are increased. The authors explain in detail the nature of the various solutions to a particular problem but, in most cases, the derivations of these solutions from the basic equations and boundary conditions have been omitted leaving the non-specialist reader to discover why such a form of solution was chosen. A rather more detailed discussion of the involved field of fracture criteria would also have been of value. For a monograph of this size the price seems excessive.

\section{A. H. England}

\section{PERIODATE OXIDATIONS}

\section{Periodate Oxidation of Diol and other Functional Groups}

Analytical and Structural Applications. By Glenn Dryhurst. (Moncgraphs in Organic Functional Group Analysis, Vol. 2.) Pp. xii + 191. (Pergamon: Oxford, London and New York, March 1970.) 70s; \$9.50.

IN spite of the recent proliferation of serialized reviews and monographs on topics in organic chemistry, resourceful editors continue to find aspects of the subject which are relatively neglected and in so doing they provide an invaluable service. The series on "Organic Functional Group Analysis" is certainly to be welcomed, and the subject and the timing of the second monograph are appropriate, but a well conceived plan has not yielded a satisfactory product. The author has, however, brought together a large amount of previously uncorrelated material and on this account will earn the gratitude of many analytical and carbohydrate chemists.

The presentation appears in places like an "Annual Report" with catalogued and insufficiently critical abstracts of the literature, and at least one transparent error in an original publication has been reproduced. While this approach could be justifiably defended, the defence is weakened appreciably by the fact that only about fifteen of the 500 references cited (with particular disregard for accurate reproduction of authors's names) are dated 1966 or later. Significant results published as long as three years ago--for example, on the oxidation of glycosides in dimethyl sulphoxide--are not included.

After a historical introduction the preparation and 